\title{
Nuclear Fuel Cycle Evaluation and Real Options
}

\author{
L. Havlíček
}

The first part of this paper describes the nuclear fuel cycle. It is divided into three parts. The first part, called Front-End, covers all activities connected with fuel procurement and fabrication. The middle part of the cycle includes fuel reload design activities and the operation of the fuel in the reactor. Back-End comprises all activities ensuring safe separation of spent fuel and radioactive waste from the environment. The individual stages of the fuel cycle are strongly interrelated. Overall economic optimization is very difficult. Generally, NPV is used for an economic evaluation in the nuclear fuel cycle. However the high volatility of uranium prices in the Front-End, and the large uncertainty of both economic and technical parameters in the Back-End, make the use of NPV difficult. The real option method is able to evaluate the value added by flexibility of decision making by a company under conditions of uncertainty. The possibility of applying this method to the nuclear fuel cycle evaluation is studied.

Keywords: Nuclear fuel cycle, front-end, back-end, economic evaluation, evaluation methods, NPV, uncertainty, flexibility, real options.

\section{Nuclear Fuel Cycle}

The nuclear fuel cycle consists in principle of three basic parts. The first part is usually called the "Front-End", and includes all activities connected with procurement of nuclear material and services, production of nuclear fuel and its transportation to the NPP site. The second part of the nuclear fuel cycle is normally defined as use of the nuclear fuel in the reactor. It is generally referred to as "Middle" or "In-Core". For all subsequent manipulations and operations with used ("spent") fuel the term "Back-End" of the nuclear fuel cycle is used. There are different points of view on the nuclear fuel cycle. In this paper I will look at it mainly from the position of a nuclear power plant operator.

\subsection{Front-End}

The Front-End nuclear fuel cycle starts with mining of uranium ore. Then uranium in the form of concentrates is purified and transformed into uranium hexafluoride in a conversion plant (uranium conversion). In light water reactors it is necessary to increase the proportion of fissionable isotope ${ }^{235} \mathrm{U}$ (in nature only $0.711 \%$ ) to $3-5 \%$ in the nuclear fuel. Such isotopic enrichment is based on separating the initial quantity of uranium hexafluoride in gaseous form into the enriched and the depleted streams using a slightly lower mass of the $\mathrm{UF}_{6}$ molecule of ${ }^{235} \mathrm{U}$ then ${ }^{238} \mathrm{U}$ (uranium enrichment). The enrichment plant uses diffusion or centrifuge technology. Uranium hexafluoride also has very convenient properties for phase transformation. Easy transition between solid, gaseous and liquid phases enables simple and safe filling, handling and transportation of uranium hexafluoride. Finally, in the fabrication plant uranium hexafluoride is re-converted into $\mathrm{UO}_{2}$. Fuel pellets are pressed from $\mathrm{UO}_{2}$ powder, sintered, braced and stacked into fuel elements (zirconium tubes). The completed fuel (fresh fuel) assembly is an array of fuel elements fixed together via the top and bottom nozzle and several spacer grids. The fresh fuel is then transported in dedicated containers to the NPP site and stored in fresh fuel storage.

\subsection{Middle part}

As fissionable isotopes are gradually consumed in the reactor core, due to their depletion a chain reaction is not longer sustainable under safe conditions. Reactor operation must be halted. During outage the most depleted nuclear fuel is replaced by fresh fuel and the quantity of fissionable isotopes in the reactor (core reactivity) is increased again. In fact, the reactivity in the core must be excessive at the beginning of the cycle in order to enable 12 months or longer operation of the reactor. Such excessive reactivity must be compensated at the beginning of the reactor cycle by burnable absorbers integrated in the fuel (gadolinium or boron based), control clusters and boron acid concentration in the primary circuit. In case of a 12-month cycle between two refueling outages, $1 / 4-1 / 5$ of the fuel inventory in the reactor core is changed. In the case of a 18 -month cycle, roughly $1 / 3$ of the fuel is reloaded, and in case of a 24-month cycle about $1 / 2$ is changed. Partly used fuel remaining in the core is repositioned and / or rotated.

Important aspects of the Middle part of the cycle are fuel design and loading pattern calculation. The basic input for this calculation is the required operation of the NPP unit (defined by the planned use of the nuclear unit within the operator's power plant portfolio) taking into account the current depletion of the fuel in the core. The design optimization sets the number of fuel assemblies to be reloaded and their enrichments, including its radial and axial profiling. The necessary content of burnable absorbers is also calculated. The loading pattern sets the position of each fuel assembly in the reactor core.

\subsection{Back-End}

The Back-End of the nuclear fuel cycle includes all operations which ensure that highly radioactive, toxic and heat releasing spent fuel is safely separated from the environment. It starts with storage of spent fuel in the pool in the reactor building. The fuel is stored until the residual heat is decreased to a level that enables further handling of the spent fuel. The capacity of the spent fuel pool is limited, and is usually suffi- 
cient only for a few years of operation. The spent fuel therefore must be transferred to some outside storage facility.

There are two basic strategies for the Back-End of the fuel cycle:

- Open cycle - the spent fuel is stored in long-term storage and then disposed of in a deep geological repository.

- Closed cycle - the spent fuel is reprocessed (the nuclear material is separated from the construction material and the products of fission reaction, and is re-used in the new fuel), and only vitrified radioactive waste is disposed of in a deep geological repository (smaller in size than for open cycle).

Treatment of radioactive waste generated during the operation of a nuclear power plant and during storage, reprocessing or other treatment of spent fuel and during decommissioning of nuclear facilities (the nuclear power plant itself, storage, reprocessing facility) is an integral part of the Back-End of the fuel cycle.

\section{Economic optimization in the nuclear fuel cycle}

All three stages of the nuclear fuel cycle are strongly interconnected and mutually dependent. The design parameters of the fuel will have a major effect on the procurement costs in the Front-End. Such procurement costs will be proportional to the heat generated in the reactor allocated to the total NPP costs during use of the fuel in the reactor. At the same time, the fuel burn-up and its power history in the core will determine the properties of the spent fuel. The resulting residual heat, the activity, the toxicity and the total quantity of such fuel will strongly influence the Back-End fuel costs. These future costs must be estimated with a sufficient degree of reliability and allocated to the unit of production generated during the lifetime of an NPP.

The above-mentioned relations illustrate the importance, and at the same time the difficulty of such complex optimization (which would take into account all three stages of the fuel cycle).

The basic assumption for optimal use of nuclear fuel is the best possible use of the neutrons produced by the chain reaction in the reactor core. Because there is an upper limit of $5 \%$ on the enrichment level used in fresh fuel (an administratively imposed limit aimed at preventing misuse of nuclear material for military purposes), the total theoretical reactivity in fresh fuel is limited. At the same time, there is another limit on the amount of reactivity loaded into reactor in fresh fuel during an outage, as the power distribution curve in the reactor core must fulfill certain conditions during the whole cycle. With the use of burnable absorbers reactivity can be temporarily suppressed but at an additional cost.

As a result, optimal designs of fresh fuel have been developed for individual reactors with the aim of making maximum use of the fuel in the reactor. Progress in the alloys used for structural fuel materials has enabled this. However such optimized fuels achieve very high burn-up, and are subjected to very harsh conditions in the core over a long period of time. The number of fuel element failures and the release of radioactivity into the primary circuit coolant has been rising with increasing fuel burn-up. This leads to increased NPP operational costs and to higher production of waste for treatment and disposal. Greater burn-up of spent fuel also means higher residual heat. Disposal containers must then be spaced further apart. This implies a larger geological repository and higher Back-End costs. Optimization only in one part of the nuclear fuel cycle can thus have an adverse effect on other parts of the fuel cycle.

It is necessary to consider that the fuel cycle is only one aspect of NPP operation. It is sometimes necessary to compromise fuel cycle effectiveness due to:

- malfunctioning of other technological systems in the power plant (operation on lower than nominal parameters or shortened cycles - not all reactivity in the fuel is used as planned and paid for),

- coordination of operation and outages of different units (not only nuclear) in the fleet of an operator (suboptimal fuel use as above),

- business opportunities - the price of electricity can motivate an operator to maximize generation despite higher specific fuel costs (this is the case of 18 and 24-month NPP cycles, fuel use and also spent fuel creation is therefore optimal for a 12-month cycle).

There is an important difference between the Front-End and Middle fuel cycle on the one hand and the Back-End, on the other. The first two stages are fully controlled by the operator. All decisions are solely at the operator's discretion. However in nuclear fuel Back-End some activities are controlled by other entities. In the Czech Republic, the state is responsible for disposal of radioactive waste, while in some countries the responsibility lies with a company founded jointly by several nuclear waste producers.

An operator and the other subject (e.g. the State) must to some degree coordinate their activities. It is questionable how the consent should be achieved between these subjects in the case of a major change in strategy (e.g. transition from an open to a closed Back-End fuel cycle) and how such important decisions will be introduced into the future repository cost estimation system and recalculated to create a new fund during NPP operation for future costs of spent fuel and waste disposal.

This review indicates that optimization over the whole fuel cycle is very difficult. In practice, some parameters must be set as fixed input values in order to decrease the number of variables to a reasonable level. In real life, fuel cycle lengths are imposed by power plant fleet outage scheduling. Consequently fuel design is optimized, and one of the criteria is to minimize the number of fresh fuel assemblies in a fuel reload batch. Other criteria are minimization of the neutron flux to the reactor vessel (lower costs for future decommissioning of the NPP, and slower ageing of the reactor vessel). More reliable fuel design leads to lower production of radioactive waste and costs for processing and disposing of them.

Currently this is a limiting factor for optimization in the nuclear fuel cycle. More detailed optimizations are performed separately in individual stages of the nuclear fuel cycle, but there is no interrelation of such analyses. Nowadays even power companies are preoccupied by short term financial results and capitalization on the stock exchange. Strategic 
long-term analysis does not fit under streamlining and cost cutting tendencies.

\section{Traditionally used analytical tools}

The basic analytical concept for financial evaluation in the nuclear fuel cycle is the Net Present Value (NPV) concept. As the analyzed options in most cases represent the same amount of generated (and sold) electricity, for NPV only costs are considered in the calculations.

\subsection{Front-End analyses}

The major uncertainty in the Front-End is the price of uranium. Following a long period of stability this price has been highly volatile for several years. The price of nuclear services (conversion, enrichment and fabrication) has been increasing steadily and is more predictable. As long-term contracts are used to procure nuclear materials and services (5-15 years), and the procurement chain for a reload batch (from mining of raw uranium to the completed fuel) is roughly 2 years, the cost of capital can be predicted reasonably. Plain NPV complemented by scenarios and sensitivity is used. For cycle $X$ of the reactor calculation of Front-End costs the following formula can be used:

$$
\mathrm{NPV}_{X}=\sum_{t=-N}^{t=M}\left(C P_{t}+F A_{t}\right) \cdot r^{-t},
$$

where $C P_{t}$ is cost of procurement in year $t$, $F A$ is fuel amortization in year $t$,

$N$ is year of the first expenditure for nuclear fuel, $M$ is year of full amortization of the fuel.

\subsection{Back-End analyses}

The Back-End period lasts at least 50-100 years. It is logical that the uncertainty is much higher than in the Front-End part of the nuclear fuel cycle. The high degree of uncertainty is connected with estimating the spent fuel and the radioactive waste storage and disposal. The actual future technologies, time schedules and waste volumes can vary substantially from those currently considered. It is difficult to predict economic parameters like inflation, interest rates and rates of return on invested Back-End liability funds over such a long period.

The most important task for Back-End analyses is to determine the rate of accumulation of financial funds (e.g. in CZK per MWh generated) during NPP operation in order to cover fully all future costs connected with nuclear liabilities (waste and spent fuel storage and disposal, NPP decommissioning). Using the traditional NPV approach, a simplified rate (constant over NPP lifetime) can be calculated in the following way:

$$
\text { rate }=\frac{\sum_{t=1-N}^{t=M} C_{t} \cdot r^{-t}}{\sum_{t=1}^{t=T} E_{t} \cdot r^{-t}},
$$

where $C_{t}$ is Back-End cost in year $t$,

$E_{t}$ is electricity generated in year $t$,

$r$ is risk free real interest rate,

$T$ is year of NPP closure,
$N$ is year $r$ of the first expenditure for nuclear fuel, $M$ is year of last Back-End cost.

In most cases, this type of NPV is used in analyses, complemented by sensitivity analysis, scenarios and trees. However a probabilistic approach is used in some countries. The individual input values are represented by probability distribution. Result is also represented by a probability distribution. The interpretation can be such that the operators are required to accumulate financial means at a rate corresponding to $50 \%$ probability and to provide guarantees corresponding to the difference between rate for $80 \%$ probability and $50 \%$ probability.

Due to the high degree of uncertainty and volatility, it is tempting to use the real option concept and its applicability to analyses in the nuclear fuel cycle.

\section{Concept of real options}

\subsection{What is an option}

The term "option" can be understood as any situation which gives a subject the right to postpone its decision until new or better information is available. There are two important terms related to options - uncertainty and flexibility.

Uncertainty is a term used to describe something which is not known. It is the set of options that are beyond the knowledge and power of the subject making the decision. Uncertainty can be technical or economic. Technical uncertainty is not a function of any economic parameter. It is a function of purely technical factors (unknown ore grade, fuel deterioration, geological parameters at the repository site). Technical uncertainty can be decreased by investment in research and exploration. It is wise to do this in stages. Economic uncertainty is a function of movements in economic parameters and in a particular industrial branch (e.g. commodity prices). Uncertainty can be quantified by volatility (standard deviation, variance).

Flexibility is the ability to adapt decisions in real time to a change in situation (market prices, new technology). However, flexibility does not always have a useful value for a decision making subject (Table 1).

Based on the degree of uncertainty and flexibility, the fol-

Table 1: Value of flexibility

\begin{tabular}{|c|c|c|c|}
\hline \multicolumn{2}{|c|}{} & \multicolumn{2}{|c|}{ System description } \\
\cline { 3 - 4 } & low & $\begin{array}{c}\text { Retter to } \\
\text { focus at the } \\
\text { beginning }\end{array}$ & $\begin{array}{c}\text { Flexibility too } \\
\text { expensive }\end{array}$ \\
\hline \multirow{2}{*}{ Uncertainty } & high & $\begin{array}{c}\text { Rigid system } \\
\text { too risky }\end{array}$ & $\begin{array}{c}\text { Flexibility } \\
\text { needed }\end{array}$ \\
\cline { 2 - 4 } & & & \\
\hline
\end{tabular}

lowing analytical methods are recommended (see Table 2).

This does not mean that option techniques cannot be used for low flexibility or uncertainty, it just wouldnot be effective. It would provide the same results as NPV. However, the meth- 
Table 2: Recommended analytical methods

\begin{tabular}{|c|c|c|c|}
\hline & & \multicolumn{2}{|c|}{ System description } \\
\hline & & Rigid & Flexible \\
\hline \multirow{2}{*}{ Uncertainty } & low & NPV & $\begin{array}{l}\text { Dynamic } \\
\text { methods, } \\
\text { Scenarios }\end{array}$ \\
\hline & high & $\begin{array}{c}\text { Sensitivity } \\
\text { Analysis, } \\
\text { Simulations }\end{array}$ & Real Options \\
\hline
\end{tabular}

ods can be combined. For example volatility can be simulated by the Monte Carlo method.

\subsection{Real options}

A Real Option is the right, but not the obligation, to undertake a business decision in future concerning a company's assets. The attraction of such a right depends on the value of the underlying asset. The current price of an underlying asset is the current value of future cash-flows. Exercise price is a capital investment connected with the decision. The option expiry date is not known in advance. If the option can be exercised at any time the expiry date, we speak of an "American option". If option can be exercised only at the expiry, we speak of a "European option". The risk-free interest rate is the same as risk-free interest rate used in financial options. The volatility of the underlying asset is expressed by the volatility of the future discounted cash-flows. By analogy with the theory of financial options, the option can be a "put" (to sell) or a "call" (to buy).

If NPV is the value of the project without considering the real option, then the following formula is valid:

$$
\mathrm{NPV}^{*}=\mathrm{NPV}+\text { value of the option. }
$$

Traditional NPV represents the value of the project when parameters estimated at the beginning are valid. However, the option value includes value of adaptation to the new conditions.

The most widely-used options are:

- Option to wait or to defer a project

- Option to expand or to contract a project

- Option to abandon a project

- Option to stage

- Option to shutdown and restart

- Option to switch

\section{Applicability of the real option concept in nuclear fuel cycle}

\subsection{Applicability in the Front-End}

If an operator buys nuclear materials and services to be delivered just in time for the next stage of the processing chain, there is no room for flexibility. The operator cannot adapt its procurement to the situation in the market. However, as soon as the operator creates strategic stocks of nuclear material (and today almost $100 \%$ of nuclear utilities do this), which would enable fuel to be produced without buying on the market for some time, an option to defer procurement of uranium is available. Therefore the operator can wait, follow the development of the uranium price in the market and wait for better conditions. This is the American option (uranium can be bought any time before expiry - the moment when the inventory is depleted). Procurement can be also staged. A binomial model can be used for calculating the value of the option (Fig. 1).

In Middle part of the nuclear fuel cycle I do not see possi-

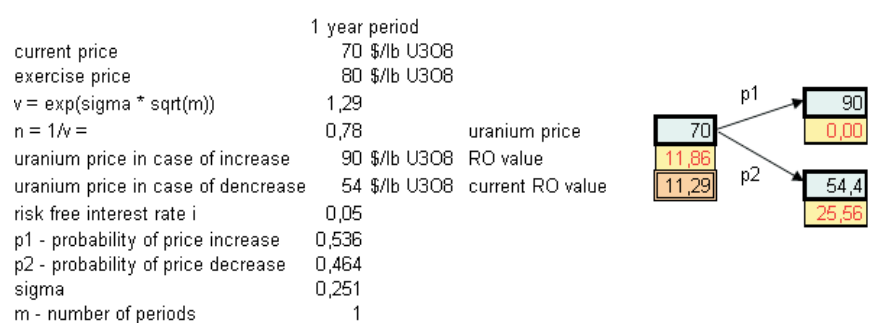

Fig. 1: Calculation of real option to defer contracting for uranium

bility of using a real option.

\subsection{Applicability in the Back-End}

On the other hand, the potential use of real options in the Back-End is wider. We can find more volatile underlying assets with at least some degree of flexibility.

If we stick with the price of uranium, we can find the first option of an NPP operator. The basic selected alternative for the Back-End is the open nuclear fuel cycle. It is assumed that all spent fuel will be disposed of in a deep geological repository after long-term storage. This alternative was selected not only for technical reasons, but mainly due to the fact that the price of uranium was low and the cost of reprocessed uranium conversion, enrichment and fabrication is much higher than the cost of freshly mined uranium. NPV preferred open fuel cycle. However if we consider the volatility of the uranium prices, and consider the option to switch from an open to a closed fuel cycle, we get a different picture.

\begin{tabular}{|c|c|}
\hline \multicolumn{2}{|c|}{ Open cycle: } \\
\hline storage & 400 USD $/ \mathrm{kgU}$ \\
\hline disposal & 900 USD $/ \mathrm{kgU}$ \\
\hline \multicolumn{2}{|c|}{ Closed cycle } \\
\hline storage & $200 \mathrm{USD} / \mathrm{kgU}$ \\
\hline reprocessing & $1500 \mathrm{USD} / \mathrm{kgU}$ \\
\hline disposal & $500 \mathrm{USD} / \mathrm{kgU}$ \\
\hline +savings on & $0 \%$ market price) \\
\hline
\end{tabular}

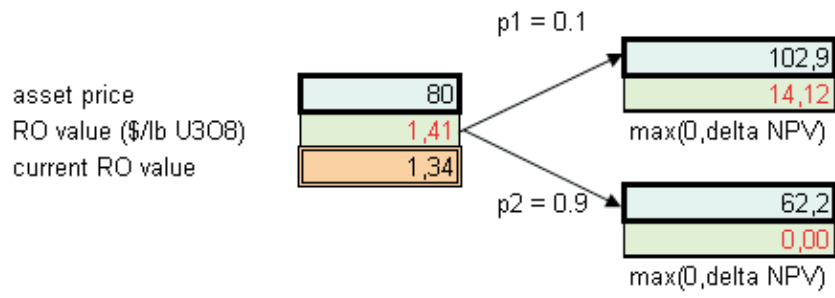

$\mathrm{NPV}^{*}=\mathrm{NPV}+\mathrm{ROvalue}=-1747.68+1.34=-1746.34$ mil. USD

Fig. 2: Real option to switch from an open to a closed fuel cycle 
Another option can be the option to expand - if a new NPP is built and the volume of spent fuel and radioactive waste is substantially changed. It is important to retain flexibility to expand the capacity of Back-End facilities. We can also imagine other real options.

\section{Conclusions}

Complex optimization of the whole fuel cycle is a very difficult task. It is necessary to set some input values in order to decrease the number of variables and make calculation viable. NPV stays the basic, for some purposes irreplaceable, analytic tool for economic evaluation in the fuel cycle. It is used for example for calculating the rate to be paid per unit of electricity generated in order to create sufficient funding for future Back-End liabilities. NPV is sometimes complemented by sensitivity analysis or probabilistic calculations. The volatility of the underlying assets and a sufficient degree of flexibility in the decisions of the NPP operator (or of the State), or flexibility of the Back-End technical solutions enables the use of real option techniques as an important support for strategic decision making.

\section{Acknowledgments}

I wish to express my gratitude to my family, to my colleagues at work, and last but not least to my teachers in the Department of Economics, Management and Humanities for their support.

\section{References}

[1] Scholleová, H.: Hodnota flexibility reálné opce. C. H. Beck pro praxi, 2007.

[2] Starý, O.: Reálné opce. A Plus, 2003.

Ing. Ladislav Havlíček

e-mail: havlicek.ladislav@seznam.cz

Dept. of Economics, Management and Humanities

Czech Technical University in Prague

Faculty of Electrical Engineering

Technická 2

16627 Prague, Czech Republic 